\title{
Identification of potential core genes and miRNAs in testicular seminoma via bioinformatics analysis
}

\author{
KAI WANG ${ }^{1}$, YUN $\mathrm{CHEN}^{1}{ }^{1}, \mathrm{ZHIHONG} \mathrm{ZHAO}^{1}$, MEIYING FENG $^{1,2}$ and SHOUQUAN ZHANG ${ }^{1}$ \\ ${ }^{1}$ Guangdong Provincial Key Laboratory of Agro-Animal Genomics and Molecular Breeding, \\ College of Animal Science, South China Agricultural University, Guangzhou, Guangdong 510642; \\ ${ }^{2}$ College of Life Sciences, Zhaoqing University, Zhaoqing, Guangdong 526061, P.R. China
}

Received January 14, 2019; Accepted June 21, 2019

DOI: $10.3892 / \mathrm{mmr} .2019 .10684$

\begin{abstract}
Testicular seminoma is one of the most common tumours in the field of urology, and its aetiology is still unclear. The aim of the present study was to identify the factors responsible for the development of testicular cancer and to investigate whether mutations in these genes were primarily congenital or acquired. To identify the key genes and miRNAs linked to testicular seminoma, as well as their potential molecular mechanisms, the GSE15220, GSE1818 and GSE59520 microarray datasets were analysed. A total of 5,195 and 1,163 differentially expressed genes (DEGs) were identified after analysing the GSE15220 and GSE1818 datasets, respectively. Among them, 287 genes were common between the two datasets. Of these, 110 were upregulated and 177 were downregulated. Five differentially expressed microRNAs (miRs; DEMs) that were downregulated in seminoma were identified after analysing the GSE59520 dataset. Following protein-protein interaction network and Gene Ontology analysis, the five nodes with the highest degrees were screened as hub genes. Among them, the high expression of hub genes, such as protein tyrosine phosphatase receptor type $\mathrm{C}$ (PTPRC), was associated with worse overall survival. We also predicted the potential target genes of the DEMs. DNA topoisomerase II $\alpha$ (TOP2A), marker of proliferation Ki-67 (MKI67), PTPRC and ubiquitin conjugating enzyme $\mathrm{E} 2 \mathrm{C}$ were associated with the PI3K/AKT and Wnt/ $\beta$-catenin signalling pathways. In addition, $h s a-m i R-650$ and $h s a-m i R-665$ were associated with the PI3K/AKT and Wnt $/ \beta$-catenin signalling pathways. Additionally, TOP $2 \mathrm{~A}$ and MKI67 were strongly associated with the target genes $h s a-m i R-650$ and $h s a-m i R-665$, respectively. We proposed that
\end{abstract}

Correspondence to: Professor Shouquan Zhang, Guangdong Provincial Key Laboratory of Agro-Animal Genomics and Molecular Breeding, College of Animal Science, South China Agricultural University, 483 Wushan Road, Tianhe, Guangzhou, Guangdong 510642, P.R. China

E-mail: sqzhang@scau.edu.cn

Key words: testicular seminoma, microarray analysis, hub genes, microRNAs, prognosis the hub genes reported in the present study may have a certain impact on cellular proliferation and migration in testicular seminoma. The roles of these hub genes in seminoma may provide novel insight to improve the diagnosis and treatment of patients with seminoma.

\section{Introduction}

The tumour of the testis is one of the most common type of tumours in the field of urology (1). Testicular tumours are divided into seminoma and non-seminoma types, the vast majority of which are seminoma; non-seminoma types are extremely rare. Testicular tumours are almost all malignant, with germ cell tumours accounting for $90-95 \%$, and non-germ cell tumours accounting for $5-10 \%$ (2). In germ cell tumours, seminoma is the most common, accounting for $40 \%$ to $50 \%$ of primary testicular tumours, followed by embryonic cancer (20-30\%) and teratoma ( 10\%) (3-5). Left and right testicular tumours of other cell types are rare. The treatment of testicular tumours is divided into single treatment, and the comprehensive treatment of surgical treatment radiation therapy and chemotherapy (6). Unfortunately, once a testicular tumour is identified, radical orchiectomy should be performed first, and then a further treatment plan should be implemented based on the pathological findings (7-10). Hence, it is urgent and necessary to explore novel therapeutic targets for the treatment of seminoma.

In the present study, we selected three gene expression datasets (GSE15220, GSE1818 and GSE59520), which were downloaded from the Gene Expression Omnibus (GEO) database, to obtain differentially expressed genes (DEGs) and differentially expressed microRNAs (DEMs) between testicular seminoma tissues and normal tissue samples. Then, functional enrichment and network analyses were applied to identify the DEGs. Subsequently, we established a protein-protein interaction (PPI) network to identify hub genes related to seminoma. The expression values of these hub genes were determined using the online database UALCAN. Survival analysis of these hub genes was performed using the online database Gene Expression Profiling Interactive Analysis (GEPIA). The potential target genes of the miRNAs were predicted by miRwalk 3.0 and screened by The Cancer Genome Atlas (TCGA) dataset. 


\section{Materials and methods}

Identification of DEGs and DEMs. Three gene expression profiles, GSE15220, GSE1818 and GSE59520, were acquired from the GEO database (https://www.ncbi.nlm.nih. gov/geo/). The array data of GSE15220 comprising 6 paired seminoma tissues, and adjacent tissues were submitted by Cheung et al (11); GSE1818 consisted of 6 paired seminoma tissues and adjacent tissues. GSE59520 consisted of 14 seminoma tissues and three normal tissues (12). DEGs were obtained from the GEO database by GEO2R analysis (http://www.ncbi. nlm.nih.gov/geo/geo2r/). Adjusted $\mathrm{P}<0.05$ and log fold-change $(\mid \log \mathrm{FCl})>2.0$ were set as the DEG cutoff criterion. Adjusted $\mathrm{P}<0.05$ and $\log \mathrm{FCl}>1.0$ were set as the DEM cutoff criterion. The common dysregulated genes between GSE1818 and GSE59520 are presented as a Venn diagram and identified using R (version 3.6.1; https://www.r-project.org/). The Search Tool for the Retrieval of Interacting Genes (STRING) database (https://string-db.org/) for annotation, visualization and integrated discovery was employed to facilitate the transition from data collection to biological analysis. Gene Ontology (GO) and Kyoto Encyclopedia of Genes and Genomes (KEGG) pathway enrichment analyses were performed using the Enrichr online tool (http://amp.pharm.mssm.edu/Enrichr/). P $<0.05$ was set as the cut off criterion. Potential target genes of the miRNAs were predicted by miRwalk 3.0 (http://mirwalk.umm.uni-heidelberg.de/) and screened using the TCGA (https://www.cancer. gov/about-nci/organization/ccg/research/structural-genomics/tcga) database.

$G O$ and KEGG pathway analyses of DEGs. GO analysis is a commonly used method for large-scale functional enrichment research; gene functions can be classified into biological process (BP), molecular function (MF) and cellular component (CC). KEGG is a widely used database that stores data on genomes, biological pathways, diseases, chemical substances and drugs. GO annotation and KEGG pathway enrichment analyses of the DEGs identified in this study were performed using Enrichr tools. $\mathrm{P}<0.05$ was considered to indicate a statistically significant difference.

Integration of the PPI network and hub gene selection. The STRING database is designed to analyse PPI information. To evaluate the potential PPI relationships, the DEGs we identified were mapped to the STRING database. The PPI pairs with a combined score of 0.4 were extracted. Subsequently, the PPI network was visualized by Cytoscape software (version 3.7.1; www.cytoscape.org/). Nodes with a higher degree of connectivity tend to be more essential in maintaining the stability of the entire network. CytoHubba (version 0.1) (13), a plugin in Cytoscape, was used to calculate the degree of each protein node. In our study, the top five genes were identified as hub genes. miRwalk (version 3.0; $\mathrm{http}: / /$ mirwalk.umm.uni-heidelberg.de/) was used to predict the potential target genes of the miRNAs identified.

Expression profiles of hub genes based on tumour histology and survival analysis. UALCAN (http://UALCAN.path.uab. edu) is a user-friendly, interactive web resource for analysing cancer transcriptome data. According to the median expression of a particular gene, the patients with testicular germ cell tumors (TGCT) were split into high and low expression groups. The overall survival (OS) of TGCT patients was evaluated using GEPIA (14). $\mathrm{P}<0.05$ was considered to indicate a statistically significant result.

\section{Results}

Identification of DEGs and DEMs. The gene expression profiles GSE15220, GSE1818 and GSE59520 were selected in this study. Based on the criteria of $\mathrm{P}<0.05$ and $\mid \log \mathrm{FCl}>2.0$, a total of 5,195 DEGs were identified from GSE15220, and 1,163 DEGS were identified from GSE1818. Among them, 287 genes were common to the two datasets (Fig. 1). Of these, 110 were upregulated, and 176 were downregulated (Fig. 2). A total of 8 DEMs that are downregulated in seminoma were identified from GSE59520. All DEGs and DEMs were identified by comparing seminoma samples with normal samples.

Functional and pathway enrichment analyses. GO function and KEGG pathway enrichment analyses for the DEGs were performed using Enrichr (Table I). The enriched GO terms were divided into $\mathrm{CC}, \mathrm{BP}$, and MF ontology terms. The results of GO analysis indicated that upregulated genes were mainly enriched in BPs, including 'regulation of the cellular macromolecule biosynthetic process', 'B cell activation', 'regulation of nucleic acid-templated transcription', and 'regulation of gene expression', while the downregulated genes were mainly involved in 'calcium-dependent cell-cell adhesion via plasma membrane cell adhesion molecules (CAMs)', 'spermatid development', 'spermatogenesis', and 'cell-cell adhesion via plasma membrane adhesion molecules'. MF analysis showed that the upregulated genes were significantly enriched in 'RNA binding', 'protein homodimerization activity', 'protein heterodimerization activity', and 'transcription regulatory region DNA binding; downregulated genes were mainly enriched in 'motor activity, microtubule motor activity', 'actin binding', and 'ATPase activity'. For CC ontology, the upregulated genes were mainly enriched in 'focal adhesion', 'phagocytic vesicle membrane', 'integral component of the luminal side of the endoplasmic reticulum membrane', and 'early endosome membrane', and the downregulated genes were mainly enriched in 'condensed nuclear chromosome', 'centromeric region', 'cytoplasmic dynein complex', 'spindle midzone', and 'focal adhesion'.

In addition, KEGG pathway analysis showed that the DEGs were mainly enriched in 'viral carcinogenesis', 'leukocyte transendothelial migration' and 'CAMs', while the downregulated genes were mainly enriched in 'amphetamine addiction', 'long-term potentiation', and 'oocyte meiosis in diabetic complications'.

PPI network construction and the analysis of hub genes. A total of 123 nodes and 269 edges were mapped in the PPI network of the identified DEGs (Fig. 3). The 5 nodes with the highest degrees, including DNA topoisomerase II $\alpha$ (TOP2A), ubiquitin conjugating enzyme $\mathrm{E} 2 \mathrm{C}(U B E 2 C)$, protein tyrosine phosphatase receptor type $\mathrm{C}(P T P R C)$, marker of proliferation Ki-67 (MKI67), and centromere protein A (CENPA), were screened as hub genes (Fig. 4, Table II). GO term enrichment 


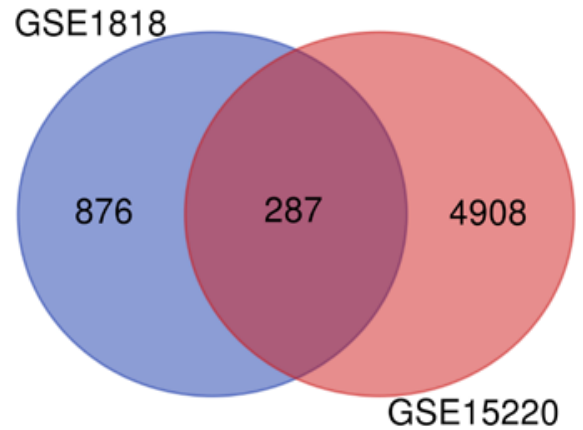

Figure 1. Venn diagram showing the intersecting genes of GSE1818 and GSE59520.

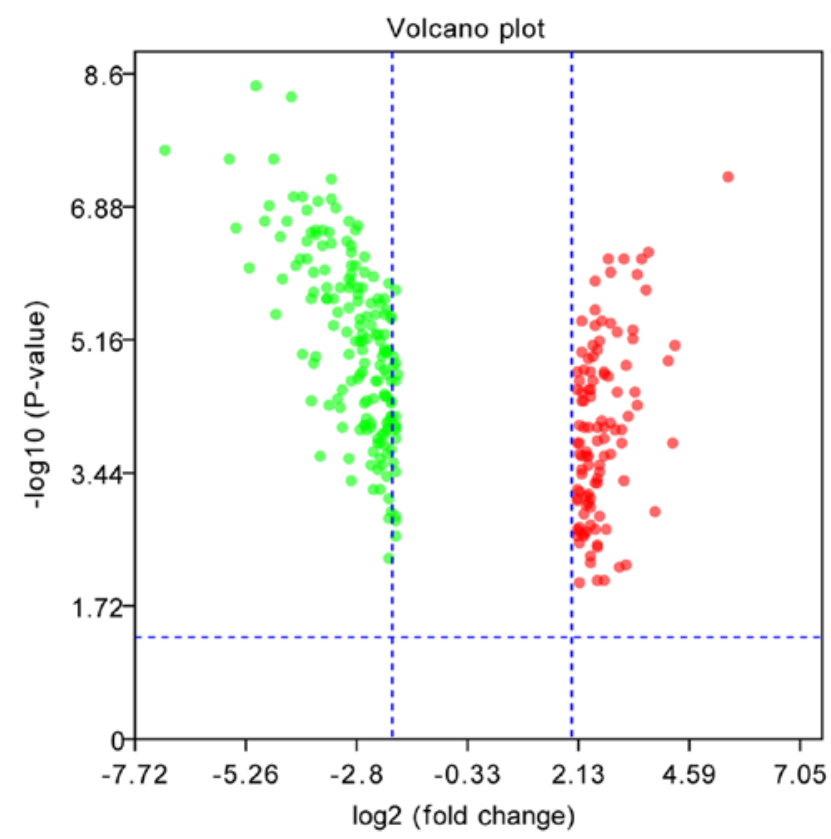

Figure 2. Volcano plot of the intersection of GSE1818 and GSE59520. Red indicates upregulated genes and green indicates downregulated genes.

analysis showed that in BPs, the genes in this module were mainly associated with "negative regulation of chromosome organization', 'regulation of metaphase/anaphase transition of cell cycle', 'regulation of cell cycle process', and 'condensed nuclear chromosome kinetochore' (Table III). The genes were significantly enriched in the 'condensed nuclear chromosome kinetochore', 'nuclear ubiquitin ligase complex' 'chromosome', and 'nucleolus' (Table III). MF analysis showed that the genes were mainly enriched in 'ubiquitin-like protein conjugating enzyme activity', 'protein kinase binding', and 'ubiquitin binding' (Table III). KEGG analysis revealed that the genes were mainly enriched in 'cell adhesion molecules', 'primary immunodeficiency', and 'Fc gamma R-mediated phagocytosis' (Table III).

Expression profiles of the hub genes and survival analysis. To investigate the expression and prognostic values of the five potential hub genes, the UALCAN bioinformatics analysis platform was used. All of the hub genes were significant (Fig. 5). We found that the high expression of these hub genes was associated with an unfavourable OS of patients with testicular seminoma by GEPIA (Fig. 6). However, because of the better prognosis of testicular cancer and few mortalities, the overexpression of only PTPRC was identified as an unfavourable prognostic overall survival in patients with seminoma.

Potential target genes of the miRNAs. To predict the potential target genes of the miRNAs, miRwalk and Cytoscape were used. miR-661 was found to be associated with PTPRC, miR-640 and miR-665 to MKI67, miR-1204 with CENPA, miR-1203 with UBE2C, miR-650 and 934 with TOP2A, and miR-1182 with TOP2A, UBE2C and MKI67 (Fig. 7).

\section{Discussion}

Although the cure and survival rates of testicular cancer are high, the main treatment methods are chemotherapy and resection (15), which presents a great burden on the patient quality of life. In addition, the incidence of seminoma is rising. The lifestyles of males in different regions vary, while the incidence rate also differs (16). It is estimated that by 2030 , there will be 65,827 new cases worldwide, an increase of 10,561 cases from 2012 (17). Although multiple approaches have reduced mortality, treatment resistance, disease relapse and treatment-derived side effects in particular, are important issues at present (18). Therefore, it is important to develop novel specific targeted therapies for the treatment of seminoma.

In our study, a total of 287 DEGs were screened, including 110 upregulated genes and 177 downregulated genes. The upregulated genes were enriched in 'viral carcinogenesis', 'leukocyte transendothelial migration' and 'CAMs', while the downregulated genes were mainly enriched in 'amphetamine addiction', 'long-term potentiation' and 'oocyte meiosis in diabetic complications'. Moreover, by constructing the PPI network, we identified five high-degree hub genes, including TOP2A, UBE2C, PTPRC, MKI67 and CENPA. Among them, all hub genes were upregulated in seminoma. Finally, we used UALCAN to analyse the expression of these hub genes; all the hub genes were reported to be significant. Then, we predicted the association between the expression of the hub genes and the prognosis of TGCT patients. Based on GEPIA, the overexpression of all hub genes was related to an unfavourable prognosis in patients with testicular cancer. Among them, we found that the overexpression of PTPRC was an unfavourable prognostic factor for patients with seminoma.

TOP2A is the molecular target of several clinically useful chemotherapeutic drugs and has been used to treat a variety of tumours, including breast cancer, prostate cancer and endometrial cancer (19). Certain studies have suggested that the overexpression of $T O P 2 A$ may be associated with the poor prognosis of these malignant diseases. For seminoma, TOP $2 A$ overexpression was associated with aggressive clinical behaviours (20). Coleman et al (21) and Sano and Shuhin (22) suggested that $T O P 2 A$ may be a marker of seminoma cell proliferation, and TOP $2 A$ was easily detected in seminoma. Additionally, studies have also revealed that $T O P 2 A$ is related to primary tumours (23-25). In prostate cancer, Labbé et al (26) found that TOP $2 A$ and EZH 2 mRNA and protein upregulation was linked to a subgroup of primary and metastatic patients with more aggressive disease, and exhibited a notable 
Table I. Significantly enriched Go terms and KEGG pathways of differentially expressed genes.

A, Upregulated

\begin{tabular}{|c|c|c|c|c|}
\hline Category & Term & Description & Count & P-value \\
\hline BP term & GO:2000112 & Regulation of cellular macromolecule biosynthetic process & 13 & 0.000044 \\
\hline BP term & GO: 0042113 & B cell activation (GO:0042113) & 4 & 0.000741 \\
\hline BP term & GO:1903506 & Regulation of nucleic acid-templated transcription & 12 & 0.000130 \\
\hline BP term & GO:0010468 & Regulation of gene expression & 15 & 0.000557 \\
\hline $\mathrm{CC}$ term & GO:0005925 & Focal adhesion & 8 & 0.000804 \\
\hline $\mathrm{CC}$ term & GO:0030670 & Phagocytic vesicle membrane & 4 & 0.000061 \\
\hline $\mathrm{CC}$ term & GO:0071556 & Integral component of luminal side of endoplasmic reticulum membrane & 3 & 0.000590 \\
\hline $\mathrm{CC}$ term & GO:0031901 & Early endosome membrane & 4 & 0.000741 \\
\hline MF term & GO:0042803 & Protein homodimerization activity & 16 & $7.14 \times 10^{7}$ \\
\hline MF term & GO:0003723 & RNA binding & 18 & 0.000550 \\
\hline MF term & GO:0046982 & Protein heterodimerization activity & 7 & 0.000675 \\
\hline MF term & GO:0044212 & Transcription regulatory region DNA binding & 10 & 0.003244 \\
\hline KEGG pathway & hsa05230 & Viral carcinogenesis & 4 & 0.026682 \\
\hline KEGG pathway & hsa04670 & Leukocyte transendothelial migration & 3 & 0.027385 \\
\hline KEGG pathway & hsa04514 & Cell adhesion molecules & 6 & 0.000135 \\
\hline
\end{tabular}

B, Downregulated

\begin{tabular}{|c|c|c|c|c|}
\hline Category & Term & Description & Count & P-value \\
\hline BP term & GO:0016339 & $\begin{array}{l}\text { Calcium-dependent cell-cell adhesion via plasma membrane cell } \\
\text { adhesion molecules }\end{array}$ & 4 & 0.000500 \\
\hline BP term & GO:0007286 & Spermatid development & 3 & 0.004178 \\
\hline BP term & GO:0007283 & Spermatogenesis & 4 & 0.047347 \\
\hline BP term & GO:0098742 & Cell-cell adhesion via plasma-membrane adhesion molecules & 5 & 0.008733 \\
\hline $\mathrm{CC}$ term & GO:0000780 & Condensed nuclear chromosome, centromeric region & 2 & 0.005635 \\
\hline $\mathrm{CC}$ term & GO:0005868 & Cytoplasmic dynein complex & 2 & 0.015846 \\
\hline $\mathrm{CC}$ term & GO:0051233 & Spindle midzone & 2 & 0.030298 \\
\hline $\mathrm{CC}$ term & GO:0005925 & Focal adhesion & 7 & 0.038965 \\
\hline MF term & GO:0003774 & Motor activity & 6 & 0.000112 \\
\hline MF term & GO:0003777 & Microtubule motor activity & 4 & 0.002046 \\
\hline MF term & GO:0003779 & Actin binding & 6 & 0.025579 \\
\hline MF term & GO:0016887 & ATPase activity & 5 & 0.034558 \\
\hline KEGG pathway & hsa00531 & Amphetamine addiction & 4 & 0.002886 \\
\hline KEGG pathway & hsa04720 & Long-term potentiation & 3 & 0.020452 \\
\hline KEGG pathway & hsa04114 & Oocyte meiosis in diabetic complication & 4 & 0.023439 \\
\hline
\end{tabular}

BP, biological process; CC, cellular component; DEG, differentially expressed gene; ECM, extracellular matrix; GO, Gene Ontology; KEGG, Kyoto Encyclopedia of Genes and Genomes; MF, molecular function.

overlap of genes involved in mitotic regulation, these results further support the hypothesis of TOP $2 A$ as a biomarker for the early identification of patients with increased metastatic potential that may benefit from adjuvant or neoadjuvant targeted therapy approaches (26). UBE2C serves as the key component in the ubiquitin proteasome system by partnering with the anaphase-promoting complex (APC/C). Upregulated $U B E 2 C$ protein expression has been reported in various types of human tumours (27). Mo et al (28) conducted an immunoassay to examine 209 breast cancer (BRCA) tissue samples and 53 normal tissue samples, and found that UBE2C is highly expressed in BRCA. Furthermore, the expression of $U B E 2 C$ was positively correlated with tumour size (24). Wang et al (29) revealed that the level of phosphorylated aurora kinase $A$ (p-AURKA) decreased markedly via the Wnt/ $\beta$-catenin and PI3K/AKT signalling pathways following the knockdown of $U B E 2 C$ with a small interfering RNA. The signalling pathway suppressed the occurrence and development of gastric cancer, and their data suggested that the activity of AURKA may be regulated by $U B E 2 C$ via modulation of the activity of 


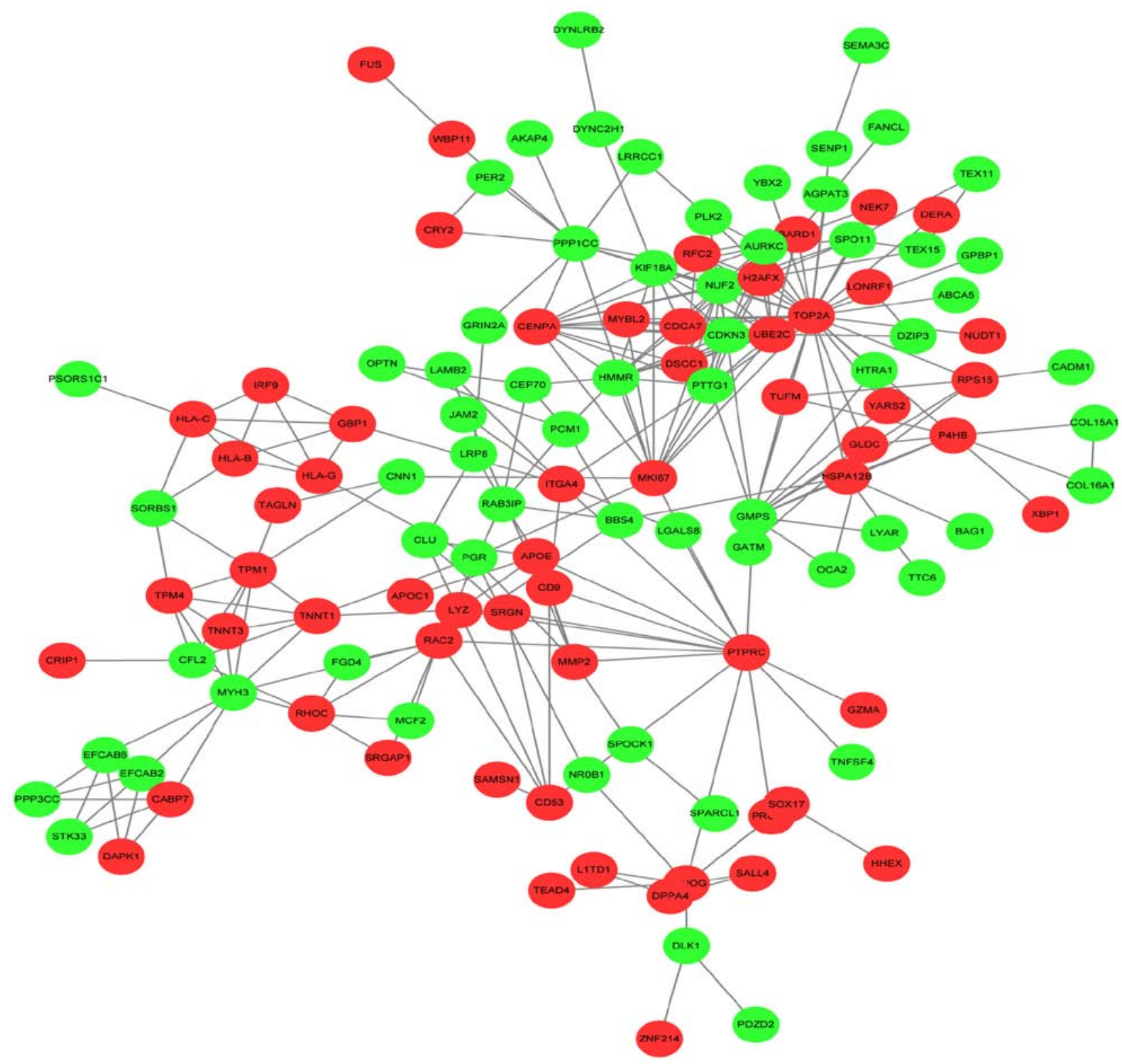

Figure 3. PPI network. A PPI network of the differentially expressed genes; red indicates the upregulated genes, while green indicates the downregulated genes. PPI, protein-protein interaction.

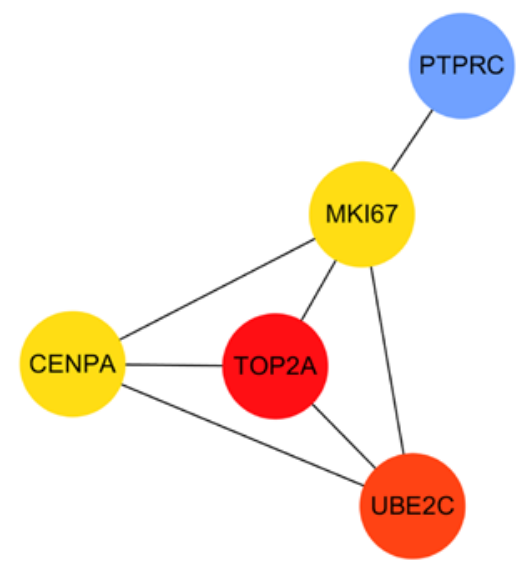

Figure 4. PPI network of a significant module. A PPI network of the top five differentially expressed genes is shown. CENPA, centromere protein A; MKI67, marker of proliferation Ki-67; PPI, protein-protein interaction; PTPRC, protein tyrosine phosphatase receptor type C; TOP2A, DNA topoisomerase II $\alpha$; UBE2C, ubiquitin conjugating enzyme E2 C.
$\mathrm{APC} / \mathrm{C}(29), U B E 2 C$ may be a novel marker in the diagnosis of gastric cancer (25); however, little is known about $U B E 2 C$ in seminoma. PTPRC is a member of the protein tyrosine phosphatase (PTP) family. PTPs are signalling molecules that regulate a variety of cellular processes, including cell growth, differentiation, mitosis, and oncogenic transformation (30). Porcu et al (31) demonstrated that the downregulation of CD45 (encoded by PTPRC) expression sensitizes T cells to cytokine stimulation, as observed by increased JAK/STAT signalling, whereas the overexpression of CD45 decreases cytokine-induced signalling. In our study, PTPRC, which may serve an important role in cytokine induction, was upregulated in seminoma. The expression of MKI67 is strongly associated with tumour cell proliferation and growth, and is widely employed in routine pathological investigations as a proliferation marker (32). It was revealed that the expression of P53 and Wnt signalling correlated with that the expression of MKI-67 in several types of cancer (33-36). Downregulated MKI67 may 
Table II. Top five hub genes with the highest degrees of connectivity.

\begin{tabular}{llc}
\hline Gene & \multicolumn{1}{c}{ Gene description } & Degree \\
\hline$T O P 2 A$ & Topoisomerase (DNA) II $\alpha 170 \mathrm{kDa}$ & 33 \\
$U B E 2 C$ & Ubiquitin-conjugating enzyme E2C & 16 \\
$P T P R C$ & Protein tyrosine phosphatase, receptor type, C & 15 \\
MKI67 & Antigen identified by monoclonal antibody Ki-67 & 13 \\
$C E N P A$ & Centromere protein A & 13
\end{tabular}

Table III. Significantly enriched GO terms and KEGG pathways of the top five hub genes.

\begin{tabular}{lllr}
\hline Category & \multicolumn{1}{c}{ Term } & \multicolumn{1}{c}{ Description } & Count \\
\hline BP term & GO:2001251 & Negative regulation of chromosome organization & 1 \\
BP term & GO:1902099 & Regulation of metaphase/anaphase transition of cell cycle & 1 \\
BP term & GO:0010564 & Regulation of cell cycle process & 0.001749 \\
CC term & GO:0000778 & Condensed nuclear chromosome kinetochore & 0.002248 \\
CC term & GO:0005730 & Nucleolus & 0.022500 \\
CC term & GO:0005694 & Chromosome & 0.002997 \\
CC term & GO:0000152 & Nuclear ubiquitin ligase complex & 0.010690 \\
MF term & GO:0061650 & Ubiquitin-like protein conjugating enzyme activity & 0.000235 \\
MF term & GO:0019198 & Transmembrane receptor protein phosphatase activity & 2 \\
MF term & GO:0043130 & Ubiquitin binding & 0.010460 \\
MF term & GO:0019901 & Protein kinase binding & 1 \\
KEGG pathway & hsa04514 & Cell adhesion molecules & 1 \\
KEGG pathway & hsa05340 & Primary immunodeficiency & 1 \\
KEGG pathway & hsa04666 & Fc gamma R-mediated phagocytosis & 0.005488 \\
\hline
\end{tabular}

BP, biological process; CC, cellular component; DEG, differentially expressed gene; ECM, extracellular matrix; GO, Gene Ontology; hsa, homo sapiens; KEGG, Kyoto Encyclopedia of Genes and Genomes; MF, molecular function.

be involved in cancer; in our study, it was downregulated in seminoma. Five pivotal genes detected in this study have been reported to be overexpressed in various human cancers, are associated with their prognosis and are significantly expressed in testicular seminoma tissues; but no significant difference in prognosis was reported. The role of these genes in TGCT is unclear; thus, further investigation is required.

Compared with normal testis samples, 11 DEMs were acquired in GSE59520 in seminoma samples. In the present study, hsa-miR-1182 was downregulated in seminoma samples. Zhou et al (37) reported that hsa-miR-1182 is dysregulated in bladder cancer tissues and cell lines, in which functional assays were then performed, the overexpression of miR-1182 significantly inhibits bladder cancer cell proliferation, colony formation and invasion $(37,38)$; however, the effects of $h s a-m i R-1182$ in seminoma are yet to be determined. Hsa-miR-650 has been reported in many cancers. For example, Zhou et al (39) observed that the expression of miR-650 in tumour tissues had a positive association with OS. Hsa-miR-650 inhibited cell growth and invasion in vitro and in vivo $(40,41)$. Furthermore, miR-650 targeted AKT2 and suppressed the activation of the AKT2/glycogen synthase kinase-3 $\beta /$ E-cadherin (39). Interestingly, Yang et al (42) suggested that phosphatase and tensin homolog/AKT signalling affects the expression of TOP $2 A$, reducing cell growth and inducing the apoptosis of human breast cancer MCF-7 cells through ATP and caspase-3 signalling pathways (42). In our study, TOP $2 A$ was identified as the target gene of $h s a-m i R-650$, and $h s a-m i R-650$ was downregulated in seminoma, while TOP $2 A$ was upregulated. Thus, we proposed that in seminoma, the downregulation of $h s a-m i R-650$ could lead to the activation of the AKT pathway, upregulating TOP $2 A$ to affect seminoma cell migration and proliferation. At present, few studies have been conducted in the investigation of hsa-miR-934. For hsa-miR-1204, Xu et al (43) revealed that $h s a-m i R-1204$ expression was significantly correlated with tumour size. The expression levels of $h s a-m i R-1204$ and glucose transporter-1 (GLUT-1) were significantly high in ovarian cancer (OC) patients (43). The expression levels of $h s a-m i R-1204$ were positively correlated with the expression levels of GLUT-1 in OC patients. Hsa-miR-1204 overexpression significantly promoted GLUT-1 expression, glucose uptake and cell proliferation. In our study, the target gene of $h s a-m i R-1204, C E N P A$, was revealed to mostly function in kinetochores and regulate cell division. The overexpression of CENPA is significantly related to colon 

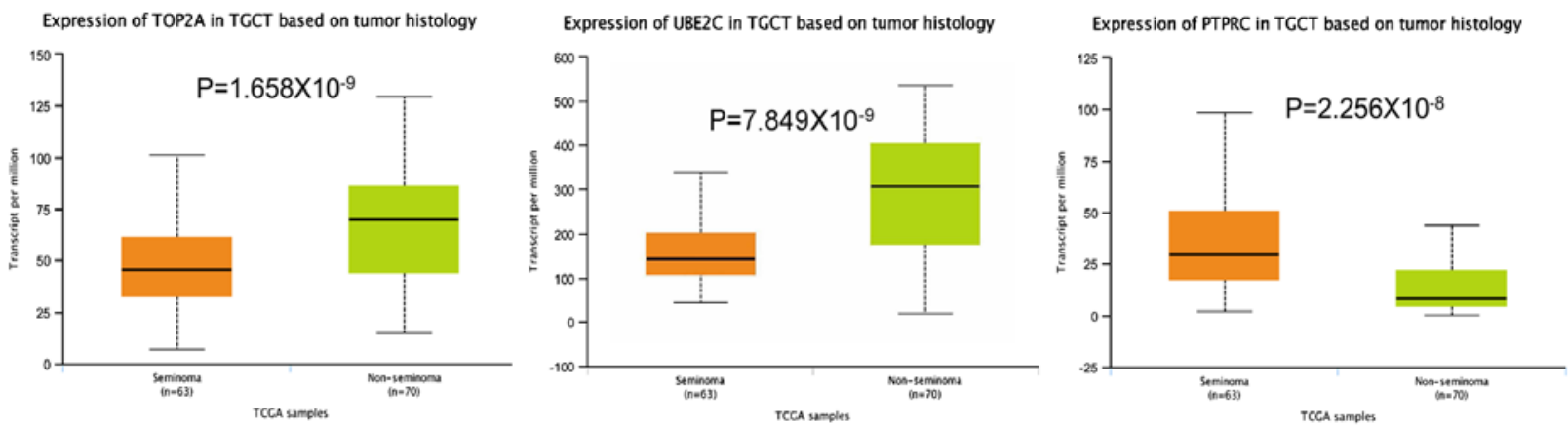

Expression of MKI67 in TCCT based on tumor histology
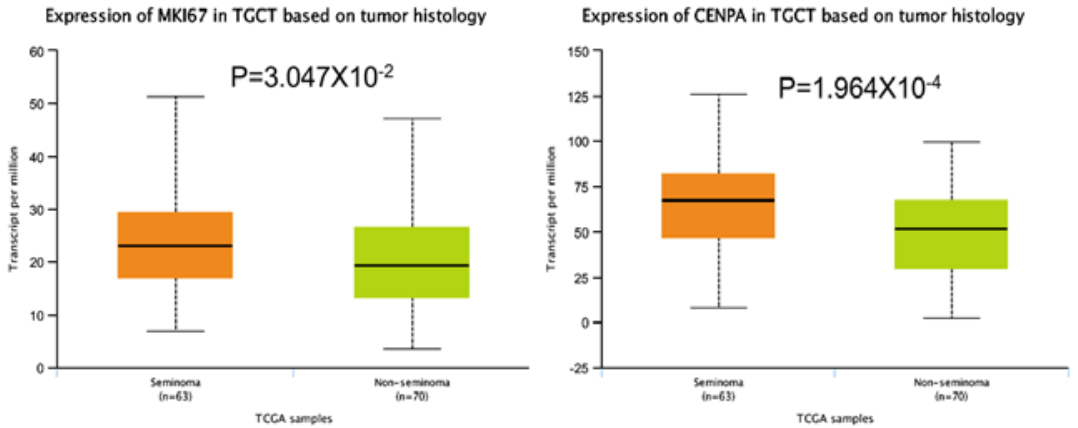

Figure 5. Expression values of the top five DEGs in seminoma and non-seminoma tissues. The horizontal lines in the figure represent maximum, upper quartile, median, lower quartile, minimum from top to bottom. CENPA, centromere protein A; MKI67, marker of proliferation Ki-67; PTPRC, protein tyrosine phosphatase receptor type C; TGCT, testicular germ cell tumor; TOP2A, DNA topoisomerase II $\alpha$; UBE2C, ubiquitin conjugating enzyme E2 C.
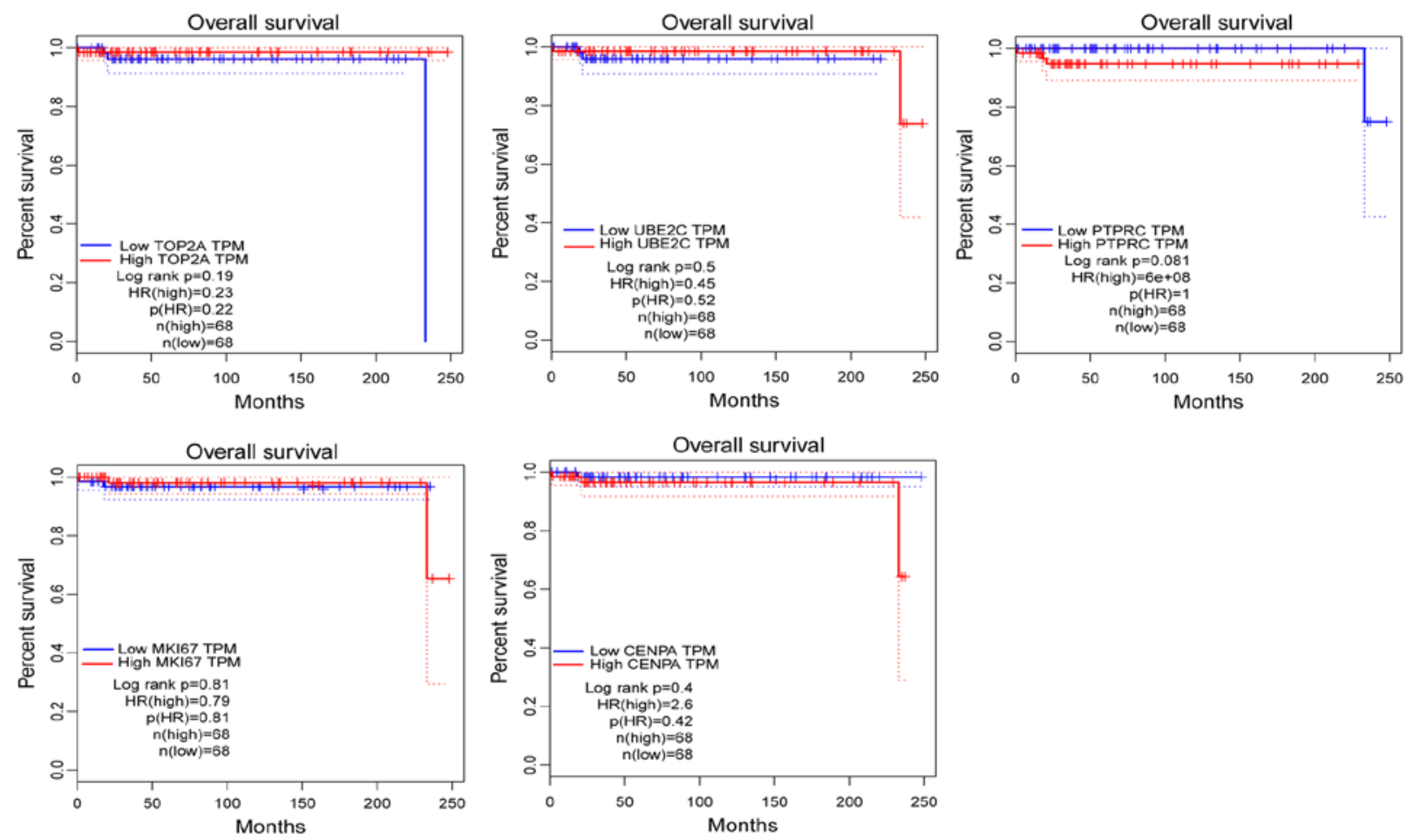

Figure 6. Gene Expression Profiling Interactive Analysis for overall survival associated with the expression of the five hub genes in patients with testicular cancer. Red line represents high expression, and blue line represents low expression. CENPA, centromere protein A; HR, hazard ratio; MKI67, marker of proliferation Ki-67; n, number of samples; PPI, protein-protein interaction; PTPRC, protein tyrosine phosphatase receptor type C; TPM, transcripts per million; TOP2A, DNA topoisomerase II $\alpha$; UBE2C, ubiquitin conjugating enzyme E2 C.

cancer and neoplastic germ cells (44,45). Hsa-miR-1204 and its target gene CENPA were both upregulated in seminoma; kinetochores are unique centromere macromolecular protein structures that attach chromosomes to the spindle for proper movement and segregation, during this process, an increasing amount of ATP is required (46), and the overexpression of $h s a-m i R-1204$ can significantly promote GLUT-1 expression, glucose uptake and ATP production (43). Thus, 


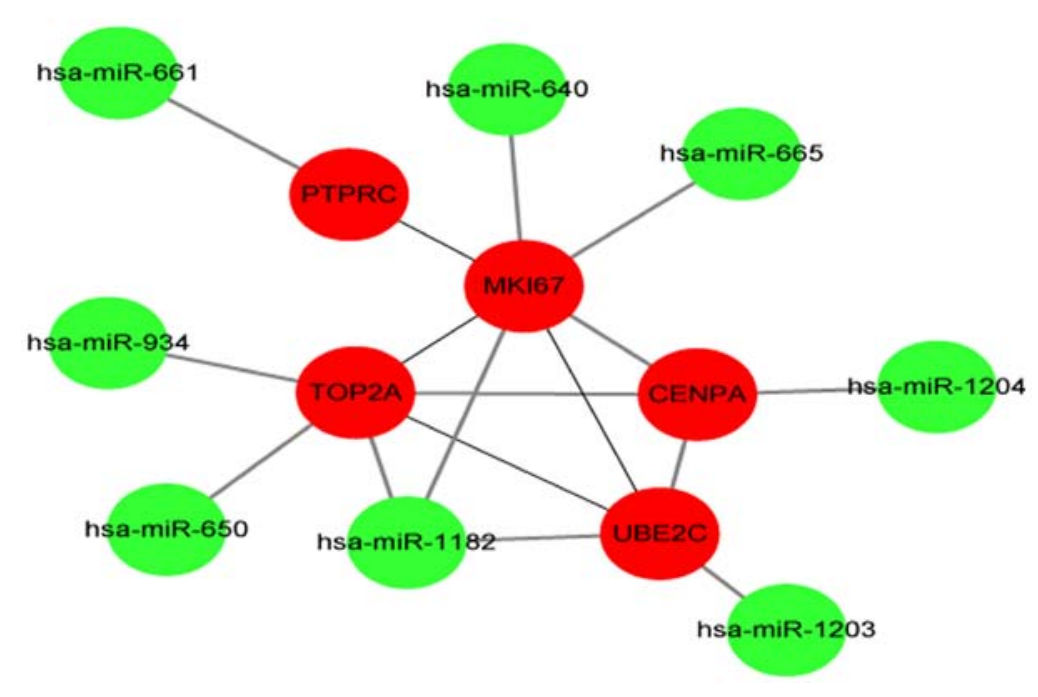

Figure 7. Protein-protein interaction network of significant miRs and their target genes. Hsa, homo sapiens; miR, microRNA; MKI67, marker of proliferation $\mathrm{Ki}-67$; PTPRC, protein tyrosine phosphatase receptor type C; TOP2A, DNA topoisomerase II $\alpha$; UBE2C, ubiquitin conjugating enzyme E2 C.

this pathway may affect the expression of CENPA; however, the association between $C E N P A$ and $h s a-m i R-1204$ requires further investigation. In addition, $h s a-m i R-661$ has been reported in non-small-cell lung cancer and OC $(47,48)$. Hoffman et al (49) found that low miR-661 expression correlates with poor outcomes in BRCA that typically express wild-type p53. In the present study, miR-661 was downregulated in seminoma. Of note, $h s a-m i R-1203$ has been reported to be dysregulated in prostate cancer, small cell carcinoma of the oesophagus and oesophageal squamous cell carcinoma (50-52). In addition, Prashad et al (53) reported that miR-665 suppresses neuroblastoma tumorigenesis by inhibiting c-MYC and suggested the potential of $h s a-m i R-665$ as an antineuroblastoma therapeutic factor. Dong et al (54) found that miR-665 was downregulated in osteosarcoma tissues compared with nontumourous tissues, and the expression of miR-665 was inversely associated with the expression of Rab23 in the osteosarcoma tissues. These results suggest that miR-665 could act as a tumour suppressor gene in the development of osteosarcoma (54); it has also been reported to be related to the Wnt/ $\beta$-catenin signalling pathway (55). In our study, miR-665 was downregulated in seminoma, and the target gene MKI67 was also associated with the Wnt/ $\beta$-catenin signalling pathway (34). For $h s a-m i R-640$, Li et al (56) demonstrated that miR-640 was downregulated in paclitaxel-resistant formalin-fixed paraffin-embedded tumour samples; however, Zhou et al (57) found that miR-640 is related to the vascular endothelial growth factor receptor 2-mTOR pathway. In the present study, hsa-miR-640 was downregulated in seminoma, and its target gene, MKI67, was also associated with mTOR (58).

In summary, TOP2A, MKI67, PTPRC and UBE2C were revealed to be potentially associated with the PI3K/AKT and Wnt/ $\beta$-catenin signalling pathways, while $h s a-m i R-650$ and $h s a-m i R-665$ were proposed to be linked the PI3K/AKT and Wnt/ $\beta$-catenin signalling pathways. Moreover, TOP2A and MKI67 were strongly associated with the target genes of $h s a-m i R-650$ and $h s a-m i R-665$, respectively.
At present, few bioinformatics analyses have been conducted to investigate DEGs and DEMs in seminoma. Of the DEGs screened in this study, only TOP2A and PTPRC have been reported in seminoma $(59,60)$, to the best of our knowledge. Additionally, we screened the differential expression of genes from array data and predicted target genes of DEMs which were proposed to act together on the PI3K/AKT and $\mathrm{Wnt} / \beta$-catenin signalling pathways with a strong correlation $(29,39)$. The PI3K/AKT and $\mathrm{Wnt} / \beta$-catenin signalling pathways participate in the growth, invasion, and migration of cancer cells in a variety of ways (61-63). TOP2A, MKI67, $P T P R C$ and $U B E 2 C$ could be used as potential diagnostic biomarkers and therapeutic molecular targets for seminoma. In addition, the main treatment methods for seminoma in clinical practice are chemotherapy and testicular resection, which undoubtedly cause great physical burden to patients, particularly in individuals aged $15-24$ years $(7,64)$. Therefore, our findings of the present study, including the genes identified, may serve as a basis for exploring gene therapy for seminoma in the future.

In this study, 287 differential genes for testicular seminoma were detected from the GEO database, and 5 pivotal genes and 8 miRNAs were screened, all of which were significantly differentially expressed in testicular cancer tissues. Among them, we predicted the target genes of the miRNAs. TOP2A, MKI67, PTPRC and UBE2C were determined to be associated with the PI3K/AKT and Wnt/ $\beta$-catenin signalling pathways, and $h s a-m i R-650$ and $h s a-m i R-665$ were associated with the PI3K/AKT and $\mathrm{Wnt} / \beta$-catenin signalling pathways. Furthermore, TOP2A and MKI67 were strongly associated with the target genes of $h s a-m i R-650$ and $h s a-m i R-665$, respectively. To the best of our knowledge, $h s a-m i R-934$ has not been investigated. There may be certain associations that are yet to be identified; the hub genes we reported could have notable impact on cell proliferation and migration in testicular seminoma. In addition, in patients with testicular seminoma, PTPRC overexpression is an unfavourable prognostic factor, and further studies are needed to verify our findings. The results of the present study suggested that TOP2A, MKI67, 
CENPA, PTPRC, UBE2C, $h s a-m i R-650, h s a-m i R-665$, hsa-miR-640, hsa-miR-1182, hsa-miR-1203, hsa-miR-661 and $h s a-m i R-1204$ may be potential targets for seminoma therapy.

\section{Acknowledgements}

Not applicable.

\section{Funding}

No funding was received.

\section{Availability of data and materials}

The datasests generated and/or analyzed during the current study are available from the Gene Expression Omnibus repository, https://www.ncbi.nlm.nih.gov/geo/query/acc. cgi?acc=GSE15220, https://www.ncbi.nlm.nih.gov/geo/query/ acc.cgi?acc=GSE1818 and https://www.ncbi.nlm.nih.gov/geo/ query/acc.cgi?acc=GSE59520.

\section{Authors' contributions}

KW and SZ made substantial contributions to the conception of the present study. KW performed the primary bioinformatics analysis and was a major contributor in writing the manuscript; YC made substantial contributions to data analysis, including the biological significance of hub genes and figure editing. ZZ and MF were involved in the interpretation of the hub genes data and edited the manuscript. All authors read and approved the final manuscript.

\section{Ethics approval and consent to participate}

Not applicable.

\section{Patient consent for publication}

Not applicable.

\section{Competing interests}

The authors declare that they have no competing interests.

\section{References}

1. Pedersen MR, Rafaelsen SR, Møller H, Vedsted P and Osther PJ: Testicular microlithiasis and testicular cancer: Review of the literature. Int Urol Nephrol 48: 1079-1086, 2016.

2. Smith ZL, Werntz RP and Eggener SE: Testicular cancer: Epidemiology, diagnosis, and management. Med Clin North Am 102: 251-264, 2018

3. Ostrowski KA and Walsh TJ: Infertility with testicular cancer. Urol Clin North Am 42: 409-420, 2015.

4. McNally RJ, Basta NO, Errington S, James PW, Norman PD, Hale JP and Pearce MS: Socioeconomic patterning in the incidence and survival of teenage and young adult men aged between 15 and 24 years diagnosed with non-seminoma testicular cancer in northern england. Urol Oncol 33: 506. e9-e14, 2015

5. Sharma P, Dhillon J and Sexton WJ: Intratubular germ cell neoplasia of the testis, bilateral testicular cancer, and aberrant histologies. Urol Clin North Am 42: 277-285, 2015.

6. Baird DC, Meyers GJ and Hu JS: Testicular cancer: Diagnosis and treatment. Am Fam Physician 97: 261-268. 2018.
7. Kamel MH, Elfaramawi M, Jadhav S, Saafan A, Raheem OA and Davis R: Insurance status and differences in treatment and survival of testicular cancer patients. Urology 87: 140-145, 2016.

8. Shen AH, Howell D, Edwards E, Warde P, Matthew A and Jones JM: The experience of patients with early-stage testicular cancer during the transition from active treatment to follow-up surveillance. Urol Oncol 34: 168.e11-e20, 2016.

9. Brand S, Williams $\mathrm{H}$ and Braybrooke J: How has early testicular cancer affected your life? A study of sexual function in men attending active surveillance for stage one testicular cancer. Eur J Oncol Nurs 19: 278-281, 2015.

10. McBride D: Surveillance is as effective as chemotherapy in stage 1 testicular cancer. ONS Connect 29: 10, 2014.

11. Cheung HH, Lee TL, Davis AJ, Taft DH, Rennert OM and Chan WY: Genome-wide DNA methylation profiling reveals novel epigenetically regulated genes and non-coding RNAs in human testicular cancer. Br J Cancer 102: 419-427, 2010.

12. Skotheim RI, Lind GE, Monni O, Nesland JM, Abeler VM, Fosså SD, Duale N, Brunborg G, Kallioniemi O, Andrews PW and Lothe RA: Differentiation of human embryonal carcinomas in vitro and in vivo reveals expression profiles relevant to normal development. Cancer Res 65: 5588-5598, 2005.

13. Chin $\mathrm{CH}$, Chen $\mathrm{SH}$, Wu HH, Ho CW, Ko MT and Lin CY: CytoHubba: Identifying hub objects and sub-networks from complex interactome. BMC Syst Biol 8 (Suppl 4): S11, 2014.

14. Tang Z, Li C, Kang B, Gao G, Li C and Zhang Z: GEPIA: A web server for cancer and normal gene expression profiling and interactive analyses. Nucleic Acids Res 45: W98-W102, 2017.

15. Bender JL, Wiljer D, To MJ, Bedard PL, Chung P, Jewett MA, Matthew A, Moore M, Warde P and Gospodarowicz M: Testicular cancer survivors' supportive care needs and use of online support: A cross-sectional survey. Support Care Cancer 20: 2737-2746, 2012.

16. Trabert B, Chen J, Devesa SS, Bray F and McGlynn KA: International patterns and trends in testicular cancer incidence, overall and by histologic subtype, 1973-2007. Andrology 3: 4-12, 2015.

17. Lobo J, Costa AL, Vilela-Salgueiro B, Rodrigues Â, Guimarães R, Cantante M, Lopes P, Antunes L, Jerónimo C and Henrique R: Testicular germ cell tumors: Revisiting a series in light of the new WHO classification and AJCC staging systems, focusing on challenges for pathologists. Hum Pathol 82: 113-124, 2018.

18. Russell SS: Testicular cancer: Overview and implications for health care providers. Urol Nurs 34: 172-176, 192, 2014.

19. Delgado JL, Hsieh CM, Chan NL and Hiasa H: Topoisomerases as anticancer targets. Biochem J 475: 373-398, 2018.

20. Panvichian R, Tantiwetrueangdet A, Angkathunyakul N and Leelaudomlipi S: TOP2A amplification and overexpression in hepatocellular carcinoma tissues. Biomed Res Int 2015: 381602, 2015.

21. Coleman LW, Perkins SL, Bronstein IB and Holden JA: Expression of DNA toposiomerase I and DNA topoisomerase II-alpha in testicular seminomas. Hum Pathol 31: 728-733, 2000.

22. Sano K and Shuhin T: A study of topoisomerase activity in human testicular cancers. Anticancer Res 15: 2117-2120, 1995.

23. Meng H, Chen R, Li W, Xu L and Xu L: Correlations of TOP2A gene aberrations and expression of topoisomerase II $\alpha$ protein and TOP2A mRNA expression in primary breast cancer: A retrospective study of 86 cases using fluorescence in situ hybridization and immunohistochemistry. Pathol Int 62: 391-399, 2012.

24. Jensen JD, Knoop A, Ewertz M and Laenkholm AV: ER, HER2, and TOP2A expression in primary tumor, synchronous axillary nodes, and asynchronous metastases in breast cancer. Breast Cancer Res Treat 132: 511-521, 2012.

25. Konecny GE, Pauletti G, Untch M, Wang HJ, Möbus V, Kuhn W, Thomssen C, Harbeck N, Wang L, Apple S, et al: Association between HER2, TOP2A, and response to anthracycline-based preoperative chemotherapy in high-risk primary breast cancer. Breast Cancer Res Treat 120: 481-489, 2010.

26. Labbé DP, Sweeney CJ, Brown M, Galbo P, Rosario S, Wadosky KM, Ku SY, Sjöström M, Alshalalfa M, Erho N, et al: TOP2A and EZH2 provide early detection of an aggressive prostate cancer subgroup. Clin Cancer Res 23: 7072-7083, 2017.

27. Xie C, Powell C, Yao M, Wu J and Dong Q: Ubiquitin-conjugating enzyme E2C: A potential cancer biomarker. Int J Biochem Cell Biol 47: 113-117, 2014

28. Mo CH, Gao L, Zhu XF, Wei KL, Zeng JJ, Chen G and Feng ZB: The clinicopathological significance of UBE2C in breast cancer: A study based on immunohistochemistry, microarray and RNA-sequencing data. Cancer Cell Int 17: 83, 2017. 
29. Wang R, Song Y, Liu X, Wang Q, Wang Y, Li L, Kang C and Zhang Q: UBE2C induces EMT through Wnt/ $\beta$-catenin and PI3K/Akt signaling pathways by regulating phosphorylation levels of Aurora-A. Int J Oncol 50: 1116-1126, 2017.

30. Vang T, Miletic AV, Arimura Y, Tautz L, Rickert RC and Mustelin T: Protein tyrosine phosphatases in autoimmunity. Annu Rev Immunol 26: 29-55, 2008.

31. Porcu M, Kleppe M, Gianfelici V, Geerdens E De Keersmaecker K, Tartaglia M, Foà R, Soulier J, Cauwelier B, Uyttebroeck A, et al: Mutation of the receptor tyrosine phosphatase PTPRC (CD45) in T-cell acute lymphoblastic leukemia. Blood 119: 4476-4479, 2012.

32. Menon SS, Guruvayoorappan C, Sakthivel KM and Rasmi RR: Ki-67 protein as a tumour proliferation marker. Clin Chim Acta 491: 39-45, 2019.

33. Yang CK, Yu TD, Han CY, Qin W, Liao XW, Yu L, Liu XG Zhu GZ, Su H, Lu SC, et al: Genome-wide association study of MKI67 expression and its clinical implications in HBV-related hepatocellular carcinoma in Southern China. Cell Physiol Biochem 42: 1342-1357, 2017.

34. Bleckmann A, Conradi LC, Menck K, Schmick NA, Schubert A, Rietkötter E, Arackal J, Middel P, Schambony A, Liersch T, et al: $\beta$-catenin-independent WNT signaling and Ki67 in contrast to the estrogen receptor status are prognostic and associated with poor prognosis in breast cancer liver metastases. Clin Exp Metastasis 33: 309-323, 2016.

35. Li LT, Jiang G, Chen Q and Zheng JN: Ki67 is a promising molecular target in the diagnosis of cancer (review). Mol Med Rep 11: 1566-1572, 2015.

36. Gallegos I, Valdevenito JP, Miranda R and Fernandez C: Immunohistochemistry expression of P53, Ki67, CD30, and CD117 and presence of clinical metastasis at diagnosis of testicular seminoma. Appl Immunohistochem Mol Morphol 19: 147-152, 2011.

37. Zhou J, Dai W and Song J: miR-1182 inhibits growth and mediates the chemosensitivity of bladder cancer by targeting hTERT. Biochem Biophys Res Commun 470: 445-452, 2016.

38. Zhang T, Zhao D, Wang Q, Yu X, Cui Y, Guo L and Lu SH: MicroRNA-1322 regulates ECRG2 allele specifically and acts as a potential biomarker in patients with esophageal squamous cell carcinoma. Mol Carcinog 52: 581-590, 2013

39. Zhou C, Cui F, Li J, Wang D, Wei Y, Wu Y, Wang J, Zhu H and Wang S: MiR-650 represses high-risk non-metastatic colorectal cancer progression via inhibition of AKT2/GSK3/ $\beta /$ E-cadherin pathway. Oncotarget 8: 49534-49547, 2017.

40. Ningning S, Libo S, Chuanbin W, Haijiang S and Qing Z: MiR-650 regulates the proliferation, migration and invasion of human oral cancer by targeting growth factor independent 1 (Gfi1). Biochimie 156: 69-78, 2019.

41. Xu X, Chen H, Zhang Q, Xu J, Shi Q and Wang M: MiR-650 inhibits proliferation, migration and invasion of rheumatoid arthritis synovial fibroblasts by targeting AKT2. Biomed Pharmacother 88: 535-541, 2017.

42. Yang Z, Liu Y, Shi C, Zhang Y, Lv R, Zhang R, Wang Q and Wang Y: Suppression of PTEN/AKT signaling decreases the expression of TUBB3 and TOP2A with subsequent inhibition of cell growth and induction of apoptosis in human breast cancer MCF-7 cells via ATP and caspase-3 signaling pathways. Oncol Rep 37: 1011-1019, 2017.

43. Xu J, Gu X, Yang X and Meng Y: MiR-1204 promotes ovarian squamous cell carcinoma growth by increasing glucose uptake. Biosci Biotechnol Biochem: 1-6, 2018 (Epub ahead of print).

44. Biermann K, Heukamp LC, Steger K, Zhou H, Franke FE, Guetgemann I, Sonnack V, Brehm R, Berg J, Bastian PJ, et al: Gene expression profiling identifies new biological markers of neoplastic germ cells. Anticancer Res 27: 3091-3100, 2007.

45. Liu R, Zhang W, Liu ZQ and Zhou HH: Associating transcriptional modules with colon cancer survival through weighted gene co-expression network analysis. Bmc Genomics 18: 361, 2017.

46. Collins CM, Malacrida B, Burke C, Kiely PA and Dunleavy EM: ATP synthase $F_{1}$ subunits recruited to centromeres by CENP-A are required for male meiosis. Nat Commun 9: 2702, 2018.
47. Liu F, Cai Y, Rong X, Chen J, Zheng D, Chen L, Zhang J, Luo R, Zhao P and Ruan J: MiR-661 promotes tumor invasion and metastasis by directly inhibiting RB1 in non small cell lung cancer. Mol Cancer 16: 122, 2017.

48. Zhu T, Yuan J, Wang Y, Gong C, Xie Y and Li H: MiR-661 contributed to cell proliferation of human ovarian cancer cells by repressing INPP5J expression. Biomed Pharmacother 75: $123-128,2015$.

49. Hoffman Y, Bublik DR, Pilpel Y and Oren M: miR-661 downregulates both Mdm2 and Mdm4 to activate p53. Cell Death Differ 21: 302-309, 2014.

50. Haldrup C, Kosaka N, Ochiya T, Borre M, Høyer S, Orntoft TF and Sorensen KD: Profiling of circulating microRNAs for prostate cancer biomarker discovery. Drug Deliv Transl Res 4: 19-30, 2014.

51. Okumura T, Shimada Y, Omura T, Hirano K, Nagata T and Tsukada K: MicroRNA profiles to predict postoperative prognosis in patients with small cell carcinoma of the esophagus. Anticancer Res 35: 719-727, 2015.

52. Okumura T, Kojima H, Miwa T, Sekine S, Hashimoto I, Hojo S, Nagata T and Shimada Y: The expression of microRNA 574-3p as a predictor of postoperative outcome in patients with esophageal squamous cell carcinoma. World J Surg Oncol 14: 228, 2016.

53. Prashad N: miR-665 targets c-MYC and HDAC8 to inhibit murine neuroblastoma cell growth. Oncotarget 9: 33186-33201, 2018.

54. Dong C, Du Q, Wang Z, Wang Y, Wu S and Wang A: MicroRNA-665 suppressed the invasion and metastasis of osteosarcoma by directly inhibiting RAB23. Am J Transl Res 8: 4975-4981, 2016 .

55. Li K, Pan J, Wang J, Liu F and Wang L: MiR-665 regulates VSMCs proliferation via targeting FGF9 and MEF2D and modulating activities of Wnt $/ \beta$-catenin signaling. Am J Transl Res 9: 4402-4414, 2017

56. Li X, Lu Y, Chen Y, Lu W and Xie X: MicroRNA profile of paclitaxel-resistant serous ovarian carcinoma based on formalin-fixed paraffin-embedded samples. Bmc Cancer 13: 216, 2013.

57. Zhou Y, Li XH, Zhang CC, Wang MJ, Xue WL, Wu DD, Ma FF, Li WW, Tao BB and Zhu YC: Hydrogen sulfide promotes angiogenesis by downregulating miR-640 via the VEGFR2/mTOR pathway. Am J Physiol Cell Physiol 310: C305-C317, 2016.

58. Yanai A, Inoue N, Yagi T, Nishimukai A, Miyagawa Y, Murase K, Imamura M, Enomoto Y, Takatsuka Y, Watanabe T, et al: Activation of mTOR/S6K But Not MAPK pathways might be associated with high Ki-67, ER(+), and HER2(-) breast cancer. Clin Breast Cancer 15: 197-203, 2015.

59. Dimov ND, Zynger DL, Luan C, Kozlowski JM and Yang XJ: Topoisomerase II alpha expression in testicular germ cell tumors. Urology 69: 955-961, 2007

60. Bo H, Cao K, Tang R, Zhang H, Gong Z, Liu Z, Liu J, Li J and Fan L: A network-based approach to identify DNA methylation and its involved molecular pathways in testicular germ cell tumors. J Cancer 10: 893-902, 2019.

61. Yang Q, Jiang W and Hou P: Emerging role of PI3K/AKT in tumor-related epigenetic regulation. Semin Cancer Biol: Apr 2, 2019 (Epub ahead of print).

62. Nusse $\mathrm{R}$ and Clevers $\mathrm{H}$ : Wnt/ $\beta$-catenin signaling, disease, and emerging therapeutic modalities. Cell 169: 985-999, 2017.

63. Fruman DA and Rommel C: PI3K and cancer: Lessons, challenges and opportunities. Nat Rev Drug Discov 13: 140-156, 2014.

64. Rajpert-De Meyts E, McGlynn KA, Okamoto K, Jewett MA and Bokemeyer C: Testicular germ cell tumours. Lancet 387: 1762-1774, 2016.

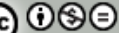

This work is licensed under a Creative Commons Attribution-NonCommercial-NoDerivatives 4.0 International (CC BY-NC-ND 4.0) License. 